\title{
Regard sur le théâtre britannique contemporain au prisme du réel
}

A look at contemporary British theater through the prism of reality

Danielle Merahi

\section{(2) OpenEdition}

\section{Journals}

Édition électronique

URL : https://journals.openedition.org/pratiques/11255

DOI : 10.4000/pratiques. 11255

ISSN : 2425-2042

Éditeur

Centre de recherche sur les médiations (CREM)

Référence électronique

Danielle Merahi, «Regard sur le théâtre britannique contemporain au prisme du réel », Pratiques [En ligne], 191-192 | 2021, mis en ligne le 15 décembre 2021, consulté le 04 janvier 2022. URL : http:// journals.openedition.org/pratiques/11255; DOI : https://doi.org/10.4000/pratiques.11255

Ce document a été généré automatiquement le 4 janvier 2022.

(c) Tous droits réservés 


\title{
Regard sur le théâtre britannique contemporain au prisme du réel
}

\author{
A look at contemporary British theater through the prism of reality \\ Danielle Merahi
}

\section{Qu'est-ce que « le réel » ? : le réel au théâtre}

1 Si c'est ce qui existe, ce qui est vrai, ce qui est avéré, alors le réel serait antinomique de la fiction. Tous les arts se confrontent au paradoxe qui consiste à retraduire une réalité en utilisant une grande diversité d'outils. Le théâtre en tant que texte de littérature, lieu de la parole et du jeu, lieu de spectacles et la scène théâtrale en tant qu'espace possible de son expression ludique grâce aux acteurs, sont des domaines privilégiés ouverts à l'imaginaire, à la création, à l'innovation et à l'expression d'une époque. Si tous les arts croisent leurs chemins, théâtre et cinéma en particulier sont des arts jumeaux et complémentaires qui permettent d'explorer les spécificités et les mutations d'une société et de son rapport au monde. Chaque période évoque son présent et son lien avec le passé selon des formes artistiques caractéristiques de leurs présents et des politiques particulières de chaque société.

2 Quant à l'artiste, il se pose comme témoin à partir des œuvres propres à son époque. Les procédés artistiques des $\mathrm{xx}^{\mathrm{e}}$ et $\mathrm{xxI}^{\mathrm{e}}$ siècles du documentaire à la fiction puisent dans le réel tout en initiant une multiplicité de formes qui permettent de «construire et expliquer le réel à partir des procédés artistiques des œuvres » (Pavis, 2019, p. 266).

Théâtre et danse tendent à collaborer et imposer leur complémentarité : la parole, le mouvement et la présence corporelle des artistes offrent une riche capacité à mixer différents arts grâce à la possible association du langage, du mouvement, du jeu. Le mot « jeu » qui implique (une ?) action est un équivalent français approximatif et réducteur du mot «performance » ou "performance art» en anglais ${ }^{1}$. Notons aussi le terme de « performer », utilisé de préférence à « actor » pour élargir le champ des possibles. 
4 Je propose d'analyser une spécificité théâtrale britannique contemporaine - Angleterre et Écosse - liée à des traditions historiques et au lien ténu qu'elles entretiennent avec le réalisme.

5 La prédilection accordée au réel, commune aux arts théâtral et cinématographique, permet de relier théâtre et cinéma contemporains à des moyens d'expression multiples telles que le documentaire et le verbatim mais aussi à bien d'autres courants artistiques, car il n'existe pas de forme canonique qui caractériserait le réalisme.

6 Traditionnellement, on peut observer que de nombreuses œuvres britanniques qui tendent à privilégier le social et le politique affichent une prédilection pour le fait divers et les histoires vraies. Le réalisme est depuis toujours le fondement de la fiction théâtrale britannique. Le théâtre, tout comme le cinéma, adopte divers modes d'écriture pour exprimer les particularismes sociétaux, la rue, le quotidien. Il s'agit de regarder, raconter, représenter... autrement. C'est-à-dire choisir ses terrains de prédilection, ses conventions d'écriture et leurs expressions scéniques. De possibles interactions permettent des lectures multiples d'histoires politiques, de faits divers du quotidien, d'articles de presse pour convoquer la mémoire collective. Les théâtres du réel favorisent le témoignage, l'exploration du passé pour éclairer le présent, la confrontation d'expériences multiples, la possibilité de rencontres.

7 L'expérience de la scène sans cesse enrichie de propositions artistiques nouvelles, le rapport à l'image (corps et décors) dans les mouvances contemporaines (body art, performance...) permettent de renouveler et enrichir les propositions et réalisations artistiques.

\section{Réel et réalité}

$8 \quad$ Faut-il défendre le droit à la subjectivité dans l'expression artistique ? « Pour moi ce qui est réel se concrétise en une chose réelle, ici et maintenant. Ce n'est pas la réalité à proprement parler mais une seconde réalité qui a été transformée et que j'ai rendue subjective en m'y confrontant.» Cette définition est affichée par le collectif Provoke lors d'une exposition de photos en décembre 2016 à Paris ${ }^{2}$. La réalité seconde qui est celle de l'artiste passe par le filtre de l'art. Le contexte historique pose la base de la réflexion à la lumière du passé et permet l'élaboration de formes spécifiques sans cesse renouvelées.

Provoke, collectif japonais militant, prend forme comme une explication et une interprétation subjective des courants de l'art japonais et de la société japonaise à un moment d'effondrement et de renouveau historiques, à la fin des années 1960 et au début des années 1970. Pour les artistes activistes de ce mouvement, le but est de mettre en valeur et critiquer les mythologies de la vie moderne en utilisant des graphismes innovants et des matériaux « médiocres " par provocation. Ils revendiquent une photographie de protestation, d'avant-garde et de théorie critique de la fin des années 1960 et du début des années 1970. Ce regard et cet engagement particuliers sur la création artistique font écho à la recherche artistique contemporaine européenne en ce qui concerne ses choix esthétiques, thématiques, narratifs plus généralement. 


\section{Réel et réalisme}

10 Dans sa confrontation avec le réel, l'expression dramatique pose singulièrement les rapports vérité-invention lorsque l'actualité nourrit la fiction et impose le réel social, historique et politique à l'art. Chaque époque aborde l'art dans un contexte spécifique qui est par essence géographique, sociétal et politique.

11 Il existe dans la tradition britannique théâtrale une spécificité qui consiste à privilégier un ancrage dans le réel. Cependant le réel n'est pas inévitablement traité de manière réaliste. La période contemporaine se caractérise par une grande diversité de formes et de thématiques où la mixité des référents est plurielle. En Grande-Bretagne, la curiosité pour des formes singulières s'affiche sous des «étiquettes" nombreuses et variées : theatre of the real, kitchen sink drama, theatre of facts, documentary theatre, agit-prop, verbatim theatre, investigative theatre, theatre of witness, autobiographical theatre, ethnodrama... Les auteurs britanniques emblématiques des années 2000 sont les héritiers d'un passé très riche et fertile issu du politique et du documentaire.

\section{Histoires individuelles, histoires collectives}

Comment les représenter? Tout est question de perspective et de traitement. Au théâtre, de nombreuses tentatives illustrent la richesse des propositions artistiques contemporaines. Les périodes de turbulences politiques et sociales privilégient l'introspection personnelle et sociétale.

13 L'exemple de D. Hare qui s'intéresse à la crise financière de 2008 dans The Power of Yes lui permet d'interroger le vrai et le faux ainsi que les limites de l'information. En 2008, il s'empare de l'histoire de la crise financière qui s'abat sur le monde en mêlant articles journalistiques et reconstitutions de scènes fictionnelles. Il la qualifie de «Greek tragedy ».

14 Quant à A. Blythe, elle imagine un procédé pour renouveler la formule documentaire en partant de témoignages authentiques et de comptes-rendus judiciaires. La diversité du public invite à introduire des références sans cesse renouvelées. En 2011, dans London Road, elle choisit une forme radicale en travaillant à partir du mot à mot d'entretiens enregistrés (verbatim) relatifs à un fait divers concernant un tueur en série qui avait agressé des prostituées en Angleterre à Ipswich en 2006. Il s'agit de montrer les faits mis à nu pour pouvoir démontrer la réalité des crimes à partir de vrais documents. Durant six mois A. Blythe a poursuivi une enquête dans cette petite ville rurale paisible en s'appuyant sur des entretiens avec la population locale. La communauté solidaire s'est alors constituée en « Neighbourhood Watch ». Après l'arrestation du coupable, un prolongement inattendu s'est produit. Les habitants de la rue ont organisé un concours de fleurissement de London Road en accrochant des paniers de fleurs dans les rues et les jardins. L'événement a ensuite été reproduit sur scène, les habitants jouant leurs propres rôles. Ce procédé du verbatim a connu un engouement aux périodes troubles de l'histoire sociale et politique de l'Angleterre suscitant la nécessité de traiter les événements à chaud. Issues du théâtre documentaire des années 1920-1930, les techniques de collage et montage de documents réels furent utilisée par des auteurs comme P. Cheeseman, D. Hare ou A. Rickman.

D'autres options artistiques telles que la danse, la musique et le chant intègrent le paysage éclectique de la recherche contemporaine théâtrale. Ne cessent d'émerger de 
nouvelles propositions qui bouleversent la scène théâtrale et la déchargent de son académisme traditionnel. Cependant ces pionniers en quête de renouveau dans le théâtre britannique ont été longtemps entravés par une absence de subventions et une censure omniprésente jusqu'en 1968 (Mérahi, 2010, p. 201).

Dans un paysage théâtral occupé par le mainstream une nouvelle dynamique se manifeste grâce aux courants rebelles des années 1950 et les pionniers emblématiques d'un théâtre politique et populaire illustrés par quelques militants activistes: E. MacColl, J. Littlewood, J. McGrath, P. Cheeseman auxquels ont succédé les babyboomers, auteurs également inventifs et politiques tels que D. Hare ou C. Churchill. Les nouvelles vagues de "collectifs » menés par des artistes emblématiques (L. Newson) imposent un langage neuf souvent provocateur. Ainsi naissent de nouvelles formes s'appuyant sur le verbatim et le corps inventif (S. McBurney). Enfin les nouveaux venus portent un regard incisif sur nos sociétés plurielles en rapide mutation et s'interrogent sur les notions d'identité et de globalisation (D. Greig, d. tucker green).

\section{Des esthétiques plurielles}

17 En Grande-Bretagne, les nouvelles approches s'efforcent de retrouver la fonction du corps et du mouvement sur une scène accaparée par la parole durant des siècles et trop longtemps régie par la censure. Oubliés les temps où tout le théâtre élisabéthain W. Shakespeare, B. Jonson, C. Marlowe, et plus tard, J. Gay - exposaient par leurs propos véhéments la violence des conditions sociales et politiques en référence avec l'actualité. Rappelons que la censure, officiellement introduite en 1737 mais souvent détournée, va sévir jusqu'en 1968 ! Elle connaitra une sévérité particulière sous l'autorité du gouvernement de M. Thatcher. Se concrétise douloureusement le lien ténu entre la politique et l'artistique. Les problèmes des subventions resteront liés à l'Arts Council. Elles seront essentiellement distribuées selon des critères politiques et constitueront un obstacle à la liberté de création. L'attribution respectait un système tripartite fonds locaux, régionaux et nationaux contrôlés par l'Arts Council - qui privilégiait le théâtre institutionnel dans des salles conventionnelles.

Le réel, c'est aussi l'ancrage sur le terrain dans un périmètre restreint où activisme artistique et activisme politico-social se rencontrent. Depuis les années 1950, une période spécifique de créations novatrices s'inscrit dans une perspective éthique et polémique où s'expriment divers courants fortement marqués par des engagements politiques contestataires précités.

\section{Le réalisme en héritage}

Historiquement le théâtre anglais a toujours tissé des liens étroits mais variés avec le réalisme. Malgré les nombreux courants artistiques qui agitent les scènes théâtrales après la Première Guerre mondiale, l'Angleterre se préoccupe peu des mouvements avant-gardistes européens tant sur le fond que sur l'aspect formel. J. Miller alias E. MacColl (1915-1989; 1990, p. 266-267) constate: "Certaines nations ont créé un théâtre de la cruauté, un théâtre de l'absurde. Nous, nous avons créé un théâtre de la morosité et de l'ennui. Et le pire c'est que nous en sommes fiers ${ }^{3}$ ».

Néanmoins après la Seconde Guerre mondiale, des innovations liées aux mutations politiques venues d'Europe et des États-Unis finissent par s'imposer et par atteindre la 
Grande-Bretagne. Emblématiques du renouveau théâtral, E. MacColl et J. Littlewood (1935-2002), encore adolescents, parviennent à développer un théâtre résolument politique de gauche révolutionnaire. Ils déclarent dans leur manifeste : «A propertyless theatre for a propertyless class », "un théâtre sans biens pour une classe sans biens ». De fait, la dynamique et la créativité du couple amèneront des changements radicaux dans la création théâtrale. Engagé très tôt dans un théâtre de rue à tendance communiste révolutionnaire, E. MacColl s'associe dans une première période à J. Littlewood. Ils vont tenter d'influencer et de bouleverser la production britannique peu imaginative et plutôt décadente des années 1930 .

21 Par ailleurs, en Europe et aux États-Unis, le regard contestataire sévit dès le début du $\mathrm{XX}^{\mathrm{e}}$ siècle. Il est caractérisé par la remise en cause des élites auxquelles répondent les mouvements syndicaux et politiques. Le factuel et le fictionnel tendent à fusionner. Le mythe qui relierait réel avec vérité se fissure et permet de s'interroger sur la frontière entre leurs champs. Dans son ouvrage True stories? D. Paget (1990, p. 39) développe pertinemment ces problématiques qui se retrouvent également dans le cinéma et plus généralement dans l'image.

Deux attitudes coexistent : Le Reporting qui permettrait de contextualiser l'événement. Le propos serait plutôt subjectif donc centré sur l'individu victime d'un système incontrôlable. L'art se dissocierait de la réalité pour focaliser sur l'individu victime et manipulé. Le Recording qui interrogerait prioritairement le contexte social et ses répercussions sur l'individu. En prenant ses distances il prétendrait rapporter un récit objectif qui poserait la question de la place et du pouvoir de l'idéologie politique.

Dans les deux cas, le théâtre documentaire par sa position au croisement de la politique et de l'art, en s'intéressant à la place de l'homme dans la cité, induit la nécessité d'élaborer des esthétiques nouvelles qui répondraient à la multiplicité des propositions artistiques qui émergent et se développent pour exploser en 1968 et provoquer des pistes plus ou moins fécondes. Le théâtre à base documentaire implique la nécessité d'un regard critique voire subversif pour se permettre de témoigner. Il offre un miroir donc un reflet de la vie telle que les politiques et les média la renvoient.

Les créations de nombreux collectifs se développent à partir des années soixante quand des artistes activistes précurseurs s'étaient imposés en proposant des structures théâtrales et des formes innovantes dans le but de défendre des textes subversifs le plus souvent à base sociale et politique. Il est essentiel de s'arrêter sur ces auteurs et compagnies novateurs qui jalonnent les années 1970 à 1980 .

En effet, les héritiers des artistes précurseurs du début du XXe siècle s'avèreront très militants pour la promotion des politiques de gauche en raison de la diffusion des mouvements communistes et révolutionnaires émergents dans l'Europe de l'Est et du dynamisme des syndicats états-uniens. L'esprit politique de l'agitprop permet de s'emparer des esthétiques innovantes issues du théâtre de rue et du documentaire. Ils imposent un courant novateur qui perdurera jusqu'à la fin des années 1970.

Une myriade de créateurs agit localement et s'interroge sur des problèmes locaux spécifiques en les reliant à la politique conservatrice et répressive du moment. De toute évidence la période de gouvernance très conservatrice de $M$. Thatcher, Première Ministre du 4 mai 1979 au 28 novembre 1990, était peu favorable à ces manifestations artistiques et leur imposa des conditions drastiques préjudiciables à leur survie: censure, absence de financements... 
Dans ce contexte, le Storytelling theatre (Théâtre de Récit) du collectif 7:84, très actif dans les années 1970-1980, parvient à mettre en avant l'importance du travail corporel et un théâtre de récit qui pouvait s'apparenter aussi à l'agit-prop et aux spectaclesfresques d'A. Mnouchkine, spectaculaires et collectifs. Il ambitionne de s'adresser essentiellement à un public également populaire. Très admiratif du théâtre de E. MacColl et J. Littlewood et à leurs engagements politiques très affirmés à la gauche de l'échiquier politique, J. McGrath (1935-2002), intègre l'idée qu'un thème fort et des comédiens compétents, flexibles et formés à diverses pratiques théâtrales et musicales pouvaient produire un objet artistique percutant et attirer un public populaire. En 1971, il crée avec sa femme E. McLennan et son beau-frère D. McLennan la compagnie anglo-écossaise 7:84 qui rappelle que ce nom provient de statistiques révélant que $84 \%$ de la richesse de la Grande-Bretagne était possédé par $7 \%$ de la population. Ce storytelling theatre fut à l'origine de la pièce très engagée politiquement et très acclamée The Cheviot, the Stag and the Black Black Oil. Elle reste représentative de l'action de J. McGrath menée également en Écosse et en Angleterre. À partir de 1962, J. McGrath a aussi écrit pour la BBC et dirigé la plupart des épisodes de la série populaire Z-cars.

D. McLennan (1937-2002) travaillera également en collaboration avec D. Greig à Òran Mór, Glasgow, en réaction aux coupes budgétaires drastiques des années Thatcher. Ils y mirent en place un cabaret populaire qui perdure actuellement où se partagent un court spectacle accessible à tous, un "pie» et une bière. (A Play, a Pint and a Pie) perpétuant ainsi la tradition des cèilidh, veillées écossaises populaires. Leur expérience s'appuyait sur une esthétique qui cherchait à éviter le pur divertissement et encourageait la réflexion critique tout en étant joyeusement divertissant.

Localement, dans une même démarche préconisant la reconnaissance d'un théâtre populaire local de qualité (Community Theatre) se dégagent de nombreux pionniers comme P. Cheeseman dans son théâtre en rond de Stoke-on-Trent. Ces «community theatres " ancrés dans un territoire participèrent largement à un courant populaire artistique critique et revendicatif. Ils perdurent actuellement.

\section{Avatars contemporains du réel sur les scènes britanniques}

Dans les années 2000 de nouvelles tendances foisonnèrent pour donner priorité aux mots bruts et confronter le public directement comme le proposait A. Blythe et son strict verbatim theatre, bien illustré dans sa pièce London Road $^{4}$ où un fait divers est évoqué de façon à la fois journalistique et théâtrale. Le décryptage d'interviews à la base du travail était parlé ou chanté dans le respect des rapports journalistiques et policiers ainsi que des témoignages rapportés. Le jeu était le plus souvent choral et frontal. Le public était directement confronté aux questions relatives à un fait divers qui avait bouleversé toute une petite communauté : l'assassinat de prostituées par un tueur en série, S. Wright, faisant cinq victimes dans la petite ville d'Ipswich. Une partition musicale originale avait été élaborée par A.Cork d'après les témoignages disponibles et dans le respect des rythmes du langage. Les acteurs devaient respecter le texte strict des interviews que proposait A. Blythe. Ils étaient contraints à restituer par l'écoute des enregistrements de ces témoignages sans jamais se confronter à un texte écrit. La consigne était de reproduire également les éléments parasites tels que toux, bégaiements, hésitations ainsi que le rythme du langage spécifique à chaque témoin, ses intonations, son tempo. Des auteurs comme D. Hare s'emparent à chaud de 
l'actualité de sociétés en crise politique et financière. Stuff Happens (2004) rend compte de l'engagement britannique dans la guerre en Irak, The Power of Yes (2009) s'interroge sur la crise financière à la suite du crash de 2008.

31 Actuellement, les mouvements artistiques se rencontrent et se diversifient, les artistes retrouvent progressivement une autonomie de création mais ils continuent à dépendre de fonds privés. Plus ouverte sur le monde, la société du XXI ${ }^{e}$ siècle se caractérise par des possibilités accrues de mobilité et de communication. Les mutations rapides, l'accès à une information permanente et immédiate et les facilités de déplacements et de rencontres orientent les écritures actuelles et donnent la priorité à des réflexions relatives en particulier à l'identité et au cosmopolitisme. De nombreuses écritures innovantes sont diffusées, portées par de nouvelles figures remarquables. Elles définissent notre présent et s'inscrivent dans l'histoire prolifique du théâtre contemporain : E. Bond, H. Barker, S. Kane, C. Churchill, M. Crimp ...

La scène émergente présente des ouvertures dans les domaines d'une expérimentation liée à cette profusion de propositions éclectiques qui enrichissent les possibilités offertes grâce la liberté d'invention langagière et stylistique propre à la souplesse de l'écriture britannique. La mixité des langages, l'existence de lieux de diffusion atypiques, le recours aux nouvelles technologies privilégient l'invention et la création. Les nouveaux auteurs trouvent davantage de place dans des lieux ouverts à l'innovation grâce à la multiplication des lieux d'accueil du spectacle vivant et des festivals. Le West end à lui seul héberge plus de 40 théâtres et le très réputé Festival d'Édimbourg en Écosse demeurent des incontournables. De nombreuses structures affichent une activité permanente comme le National Theatre de Londres, le Wales Millenium au Pays de Galles, la Royal Shakespeare Company à Stratford-upon-Aven, le Citizen's de Glasgow.

Que l'innovation soit liée à l'écriture textuelle ou scénique, les moyens de diffusion se multiplient et permettent une visibilité accrue. Le rôle d'Internet est déterminant et devient essentiel surtout en périodes de crises quand l'accès aux structures permanentes n'est pas disponible.

Je propose de considérer plus précisément quelques exemples de travaux d'artistes qui illustrent la diversité des créations contemporaines. Il est difficile d'effectuer un choix représentatif en raison de la richesse des propositions, néanmoins certaines approches caractérisées par leur langage artistique spécifique qui engage également le politique et le social semblent particulièrement fécondes et soulèvent des questions essentielles au présent : je retiens parmi de nombreuses compagnies exemplaires quatre compagnies innovantes contemporaines: la compagnie DV8 de L. Newson qui conjugue danse et parole reliées à l'actualité des marginaux et propose un "théâtre physique " interrogeant la notion de "théatre total»; Complicite dont le directeur artistique S. McBurney_revendique un « corps inventif » alliant théâtre et danse. Quant à D. Greig, il poursuit sa recherche dont l'ambition est de créer une œuvre essentiellement axée sur la mémoire culturelle et politique en prise avec l'évolution et les modifications sociétales tandis que $d$. tucker green invente un langage original issu de la rue, de la diaspora jamaïcaine et des mouvances musicales actuelles pour privilégier une parole et une gestuelle essentiellement collectives. 


\section{Lloyd Newson et DV8 pour un Théâtre Physique}

Créé en 1986 par le chorégraphe et danseur L. Newson d'origine australienne installé à Londres depuis 1981, le collectif de danseurs DV8 souhaite échapper à l'emprise du classique encore très ancré dans la danse contemporaine des années 1980. Il s'apparente au théâtre documentaire tel que l'a défini de manière exhaustive P. Weiss en 1971 :

Le théâtre documentaire est un théâtre de reportage. Archives, documents, lettres, statistiques, études de marché, rapports de banque et de compagnies, déclarations gouvernementales, discours, entretiens, déclarations de personnalités célèbres, presse écrite ou autre, photos, films documentaires et autres documents d'actualité sont à la base de la représentation... (Weiss, 1971, p. 41). choisi en raison des nombreux films vidéo que produit la compagnie pour la présentation de leur travail, en plus des représentations sur scène ". Il joue également sur le mot anglais deviate, signifiant "être différent, sortir de sentiers battus " 5 . Les thèmes toujours ancrés dans des problématiques marginales d'actualité et leur expression sont souvent provocateurs et montrent de manière crue les déviances liées à des comportements ou pratiques marginaux. L'importance, affirme Lloyd Newson, est de travailler sur quelque chose de concret d'où l'importance du mot physical. Le dernier spectacle du collectif, Enter Achilles créé en 2018, fait référence à l'ambiance de camaraderie empreinte d'humour qu'on retrouve dans les pubs britanniques, essentiellement les plus populaires. Comme dans la plupart de leurs créations, la frontière entre danse et théâtre est particulièrement ténue. Ici la thématique, le scénario (storyline) et les personnages font référence à la camaraderie et l'humour partagés mais aussi à leur vulnérabilité qui se traduit par la violence souvent omniprésente dans un pub populaire essentiellement masculin.

La marginalité est la référence constante des œuvres présentées par la compagnie qui continue à travailler en collectif. Théâtralement, ces thématiques diverses explorent l'actualité en privilégiant la dynamique du corps et du langage. Les provocations verbales et physiques empreignent le spectacle qui peut s'apparenter à un happening et interroge à nouveau la notion de «théâtre total ». Cette mouvance lui permet d'utiliser la scène comme un outil politique en servant de porte-parole à des minorités diverses : marginaux, handicapés, hors normes (d'où le nom de la Compagnie "Deviate») auxquels il accorde la parole sans jugement, propose leur présence sur scène ou leur représentation par des comédiens-danseurs. Le désir de provoquer un choc esthétique et émotionnel par l'exploration de la marginalité sociale et psychique et de la sublimer par l'art à travers la représentation publique provoque un trouble évident chez le spectateur et interroge sur le rôle du comédien qu'il soit personnage ou son propre sujet.

L. Newson emprunte toutes les formes artistiques et use volontiers du verbatim. MSM, créé en 1993 aborde à partir d'interviews d'une cinquantaine d'hommes partageant une forme de sexualité marginale, le cottaging. Cette pratique consiste à trouver son plaisir sexuel dans les toilettes publiques. Il s'intéresse aussi au handicap et met en scène en 2004 un danseur cul-de-jatte qui pose ses questions d'ordre intime au public: «Comment tu pisses? Comment tu fais l'amour? À quoi ressemblent tes moignons? T'en veux à Dieu d'être né ?» 
39 En 2011, il focalise son propos sur le thème de l'intolérance et la liberté d'expression. Il aborde alors un sujet devenu hypersensible, la religion musulmane et cible plus spécifiquement la communauté présente en Angleterre dans son spectacle Can we talk about this?. Le texte occupe une place essentielle dans cette performance qui fut violemment controversée. Si la gestuelle était toujours très présente, le texte était également prédominant puisqu'il s'appuyait sur des entretiens avec des journalistes, des politiques, des enseignants auxquels s'ajoutaient des documents d'archives et des films. Était mise en accusation l'intolérance d'extrémistes musulmans. Le parti pris polémique prit le tour d'une provocation quand le spectacle s'acheva par cette question piégée au public: "Would all those in the hall who think they are morally superior to the Taliban raise your hands?» (Que ceux dans la salle qui pensent qu'ils sont moralement supérieurs aux Talibans lèvent la main).

Dans le thème annoncé par le programme anglais ${ }^{6}$, le mot «Islam » figurait mais fut prudemment sorti de la liste dans le texte traduit en français lors des représentations au Théâtre de la Ville à Paris ainsi que la référence aux western democracies traduites par « sociétés d'Europe occidentale » ... On ne peut évidemment pas reprocher à L. Newson son manque de courage et son engagement pour les causes ouvertes à la polémique. Cependant réduire le thème plus général de la liberté d'expression en le centrant exclusivement sur une " communauté ethnique et religieuse » souffrait d'une approche restrictive qui peut être considérée comme discutable... mais c'était certainement le but recherché.

Quant à la forme, elle répondait aux critères d'un théâtre physique qui ambitionnerait de s'apparenter à un «théâtre total » mêlant divers moyens artistiques en accordant une importance égale au texte et au corps. En introduisant du texte verbatim, la profération devant micro posait frontalement un questionnement radical sur l'actualité sociale et religieuse du fondamentalisme musulman. Les outils techniques liés au son et à l'image étaient prédominants. Le corps du performeur demeurait cependant l'outil majeur de la performance qui, comme le note le programme, traitait à chaud de thèmes d'actualité sensibles: le problème de "la liberté d'expression et de l'Islam». Ce spectacle coup-de-poing (in-yer-face!) remplissait courageusement sa fonction provocatrice en dénonçant l'intolérance religieuse d'une communauté. Les courtes séquences se composaient de scènes reconstituées qui forçaient la caricature comme celle relative à la député Labour A. Cryer buvant son thé, assise sur le dos d'un performer. Le plus souvent, à la violence des propos répondaient des images fortes de corps manipulés, agressés, projetés sur scène. La profusion des moyens techniques et les enchainements virtuoses des courtes scènes dénuées de leur contexte visaient à provoquer le public et l'encourager à pousser un cri d'indignation. Les moyens techniques tels que projections sur quatre écrans, bande son omniprésente, micros déréalisant les voix, acoustique sophistiquée accentuaient la tension et l'extrême brutalité des séquences. L'efficacité était indéniable mais l'abondance des moyens pouvait créer une certaine saturation. La référence aux performances du Living Theatre pouvait être tangible nonobstant l'absence d'improvisation et de savant désordre. La journaliste J. Mackrell note dans sa revue critique du 4 novembre 2011 à la West Yorkshire Playhouse de Leeds (Mackrell, 2011) que si les problèmes soulevés par le collectif sont incontestablement révoltants et alarmants, la dimension historique fait défaut. En effet le spectacle omet de mentionner que parallèlement les printemps arabes manquaient de soutien alors que des régimes corrompus étaient soutenus par 
l'occident et que l'invasion de l'Irak par les États-Unis avait pu favoriser la radicalisation justement dénoncée par le spectacle.

Le théâtre de L. Newson affiche clairement ses engagements dans la vie sociale et politique contemporaine. Il participe aux mutations du langage théâtral en mixant efficacement les moyens d'expression jusqu'à l'épuisement. Le rythme effréné des prises de parole dans l'urgence sont volontairement provocatrices. L'esthétique très soignée de l'image scénique ainsi que la dynamique corporelle extrême qu'il impose aux performeurs traduisent efficacement ses indignations. Le choix de privilégier cette approche émotionnelle, de provoquer le public, d'appeler à la prise de conscience par de moyens in-yer-face risque de s'inscrire dans un présent éphémère qui ne survivra pas dans la durée.

\section{Simon McBurney et le Complicite Theatre pour un théâtre total}

Pour S. McBurney, le réel est présent dans la matérialité du corps et de l'espace. C'est la présence hautement symbolique et pratique des objets qui prédomine car ils servent à voir, à être manipulés, à exprimer. En amont, le travail invisible de la représentation sur scène s'inscrit dans la "répétition »: la répétition du regard, de l'écoute, de l'image. Quand tout est fixé, ce travail appartient alors au passé mais reste perceptible peut-être dans la gestuelle, les pieds, les visages des artisans de ce travail et permet de garder la trace de ce qui a précédé quand, au moment de la représentation, les artistes jouent sur scène le présent de cette trace fugitive. C'est ainsi que S. McBurney comprend son propre travail. ${ }^{7}$

La question essentielle qui se pose est : comment reconstituer et représenter le réel ? Le travail d'approche consiste à explorer tous les moyens artistiques et techniques: théâtre, mime, jeu d'ombres, marionnettes, danse, vidéo, musique, traverser tous les champs artistiques mais aussi développer un espace sensoriel qui relie les artistes entre eux mais aussi au public. Cette forme de théâtre total qui croise les références et les expériences fait appel à tous les sens. Elle immerge le public dans un univers irrationnel construit sur les croisements et fulgurations

Répéter. C'est le mot français qui correspond de manière inexacte au mot anglais rehearsal. S. McBurney les revendique tous deux. On répète, on répète encore...Répéter, c'est espérer que quelque chose survienne, ce n'est pas l'ennemi de l'originalité et de l'inspiration, c'en est la source. La fascination des objets qui conduit son travail est née de son enfance où il accompagnait son père sur son lieu de travail au Museum of Archeology and Anthropology à Cambridge. Dès l'enfance, il s'est frotté au rituel et aux objets exposés. Derrière chaque étoffe, chaque objet un visage se dessinait. Ils portaient l'empreinte d'une vie.

Rehearsal au sens littéral c'est la herse (herce étymologiquement) quand on rake over, on râcle, on prépare le sol pour la semence. Se dessine la figure du laboureur qui passe et repasse avec sa herse pour obtenir un terreau fertile. Le geste est banal et répétitif. Ainsi nait la vie.

47 Élève de J. Lecoq dans les années 1980, à l'École Internationale de Théâtre, il fuit l'Angleterre de $\mathrm{M}$. Thatcher et tente de conjurer la mort de son père en s'établissant à Paris. L'enseignement de J. Lecoq, peu dogmatique mais respectueux de la créativité de chaque personne, est un moment essentiel pour sa reconstruction, une initiation et finalement une incitation à trouver sa "voie». Il retient de cette période 
d'apprentissage «le sens de l'observation, l'importance du corps, l'émotion du geste, la puissance expressive de la présence... » (David, 2006).

Toute la base de son travail artistique prolifique se construit grâce à la philosophie empirique de J. Lecoq qui aurait déclaré : «Le muscle qui m'intéresse le plus n'est pas celui de l'intelligence, de la voix ou du physique : c'est celui de l'imagination. " Il retiendra ce qui constitue la base de son propre travail : «Comment un mot peut créer un espace, comment user de la métaphore pour transposer une idée dans une forme » (Id.).

L'esthétique et la dramaturgie de S. McBurney sont non seulement empreintes de l'héritage de J. Lecoq mais aussi d'influences multiples provenant de l'Europe jusqu'à l'Orient. Elles peuvent être visuelles, littéraires (H. Murakami pour The Elephant Vanishes), ou personnelles comme A Minute too Late qui évoque la mort de son père. Elles peuvent aussi être issues des réminiscences d'œuvres théâtrales comme celles de S. Beckett (Endgame) ${ }^{8}$.

50 Toujours, le corps dynamique et l'objet demeurent des éléments premiers qui obligent les performers à s'éloigner du texte. Ils permettent de dévoiler son univers privé empreint de réflexion poétique, morale, philosophique. De nombreuses influences ont permis d'ouvrir son imaginaire à une infinité de possibles. Des artistes fondamentaux comme V. Meyerhold, T. Kantor, J. Grotowski, B. Brecht se profilent dans son œuvre. Le théâtre de P. Brook, la danse-théâtre néo-expressionniste de P. Bausch ou même les pantins du cabinet de curiosités de J. Nadj y laissent leurs empreintes.

51 L'écriture scénique s'affilie aux démarches propres au "théâtre physique » et " théâtre du mouvement ». Si parfois des auteurs marquent son œuvre, ils sont des tremplins qui lui permettent de repartir et poursuivre son propre chemin. Son parcours n'est jamais solitaire. Il entraine avec lui une équipe qui accepte les règles du moment. Les considérations matérielles autour de la réalisation d'un spectacle sont fréquemment les points de départ du projet artistique. Par exemple, le projet autour de Mnemonic consistait à s'interroger sur la représentation d'un corps vieux de 5000 ans qui fut retrouvé dans un glacier en 1992. La représentation littérale du cadavre sur scène était exclue, donc il fallait le représenter autrement: par les mots, par un accessoire. En répétition c'était une chaise qui le matérialisait. Donc la chaise fut utilisée. Comme une chaise cassée semblait plus évocatrice, elle fut finalement adoptée.

In Mnemonic we had to represent a 5000-year-old corpse that emerges brown and wrinkled out of a glacier in 1992. It became clear to us that any literal representation would be more than faintly ludicrous. The words alone that described his appearance were stronger than any banal prop.

But something had to stand in for its presence.

So we used a chair.

But the chair was more evocative if broken.

So we used a broken chair ${ }^{9}$ (Ainslie \& Warren-Fischer, 2010, p. 100).

\section{Perspectives nouvelles : David Greig, debbie tucker green.}

L'univers du théâtral britannique actuel confirme l'émergence d'écritures très diversifiées et de leurs transcriptions scéniques impliquant un nouveau rapport à des publics socialement divers. Ce sont aussi des auteurs qui illustrent les possibilités de mobilité qu'offre notre époque.

53 D. Greig et $d$. tucker green sont tous deux engagés dans leur recherche de formes théâtrales et de thématiques contemporaines. Ils sont, en quelque sorte, 
emblématiques d'une société qui s'est délitée et poursuit une quête de sa propre identité pour se reconstruire. La forme théâtrale - son ouverture et sa souplesse mais ses contraintes également - assumerait alors un rôle d'observatoire et témoignerait des mouvances de notre époque qui aspirerait à une identité multiple élargie plus stable, plus riche, plus sereine. Ainsi pourraient se tracer des itinéraires originaux hors des sentiers battus par la violence du monde contemporain. Ils pourraient permettre aux artistes de témoigner et d'offrir un miroir propre à faire face aux contradictions de notre époque.

D. Greig, né en 1969, est un auteur extrêmement actif et prolifique. La mobilité géographique qui caractérise sa vie et son œuvre préfigure la profusion de ses réalisations artistiques. Il est metteur en scène de ses pièces et adaptations et occasionnellement traducteur. Il peut être également directeur de théâtre et écrire pour un public élargi dans des structures officielles mais aussi marginales. Son parcours foisonnant de rencontres diverses, ses engagements artistiques et politiques l'inscrivent dans la dynamique du XX $\mathrm{X}^{\mathrm{e}}$ siècle, siècle du mouvement.

Très disert, il parle volontiers de son œuvre qui mêle fréquemment fiction et Histoire. Il parcourt aussi bien des espaces réels que des lieux imaginaires. Cependant restent omniprésents les vastes espaces géographiques, de l'Écosse au Moyen Orient. Il défend et incarne un paradoxe : appartenir à un lieu et se définir cosmopolite. Il adopte un parcours fictionnel foisonnant et diversifié si on considère sa mobilité dans l'espace géographique réel, sa liberté dans des espaces historiques revisités. Son implication militante et son approche critique en prise avec les problèmes du présent affichent la cohérence de sa direction politique. Son imaginaire fictionnel l'entraine dans des vagabondages spatiaux et temporels questionnant notre présent dont il se fait le porteparole. Son point de vue balaye cependant la sphère universelle et porte, selon P. Pavis, une « parole agissante » où « l'individu devient collectif » (Pavis, 1997).

Les vagabondages de D. Greig sont incessants et insatiables. Cette urgence traduit paradoxalement son désir compulsif d'enracinement lié à la conscience implicite qu'il n'appartient à aucun territoire. Cette particularité se traduit par l'éclectisme de son parcours artistique et de ses choix thématiques. La notion d'identité si difficile à cerner caractérise l'ensemble de son travail de dramaturge, metteur en scène et directeur de structures théâtrales. Le terme «identité » recouvre cinq nuances de sens : similitude, unité, identité personnelle, identité culturelle et propension à l'identification (Baudry \& Juchs, 2008, p. 155). C'est pourquoi raconter des histoires, c'est aussi raconter l'Histoire. C'est retrouver une langue qui soit épique, poétique aussi bien que populaire. C'est redécouvrir ou refonder un lieu pour l'habiter.

Quand D. Greig s'empare de l'histoire écossaise, il prend plaisir à manipuler les possibilités d'une langue riche et complexe qui a traversé les siècles et manifeste sa présence bien ancrée dans un territoire, surtout dans le nord de l'Écosse. Ses parcours sont empreints des paysages traversés et de lieux multiples depuis les iles d'Atlantique nord, San Diego, Kyoto, Damas, une chambre d'hôtel, un aéroport, jusqu'à une navette spatiale... L'aspect narratif de son théâtre est prédominant. Il raconte des histoires, des aventures humaines et des fables politico-sociales. Implantées dans la réalité sociale et politique, ses thématiques se déclinent au présent. Il pose sur l'actualité un regard souvent iconoclaste et privilégie l'exploration de l'immédiateté de l'information pour interroger plus généralement l'histoire contemporaine. Damascus pointe la difficulté de l'échange interpersonnel quand on appartient à des univers géographiques et socio- 
culturels éloignés. Dans sa pièce Dunsinane, le Macbeth shakespearien est revisité pour s'interroger sur la légitimité d'une invasion à la lumière d'une sordide actualité - celle de l'invasion de l'Irak. Dans The Events, il propose un dialogue fictif pour tenter de comprendre un acte criminel politique insensé très réel : l'attaque terroriste perpétrée par le Norvégien A. B. Breivik en 2011.

La langue de D. Greig se colore d'une grande variété de tons. Chaque texte possède sa propre voix qui renvoie au genre littéraire abordé, de l'intime à l'épique et la forme s'adapte au format théâtral choisi. La thématique, particulièrement variée, exprime les problèmes liés à l'actualité et à l'identité. Elle est flexible et sert le propos qui est le plus souvent sociétal et politique.

d. tucker green, née à Londres d'une famille d'origine caribéenne ou British West Indian (Olusoga, 2017, p. 428), est une artiste indépendante affichant sa propre culture noire et ses convictions féministes. Son expérience comme régisseuse durant 10 ans lui ouvre des perspectives multiples relativement à la scénographie qui occupe une large place dans la mise en œuvre de ses spectacles. Ses compétences techniques sont indissociables de son écriture théâtrale et cinématographique. Elle privilégie des formes brèves et percutantes. Auteure, directrice d'acteurs, scénographe, elle coordonne l'ensemble de ses productions. Sa maitrise de la caméra lui permet également de produire des images sophistiquées très incisives.

Dans la mouvance actuelle du "Black drama », les dramaturges issus de l'émigration noire réclament leur place pour exprimer les revendications et les spécificités des communautés dans une société désormais multiraciale. Le temps de la diaspora intégrant une culture étrangère est révolu. Ils/elles affirment l'expérience d'une perspective culturelle élargie et proposent la création d'un nouvel arsenal linguistique qui bousculerait la syntaxe, introduirait un langage et un discours qui se confronteraient aux « impasses du langage » (Peacock, 2015, p. 154)

61 Dans ce contexte très combatif, $d$.tucker green occupe une place essentielle. Son théâtre aborde des thèmes a priori spécifiques des sociétés marginales sousprolétariennes pour élargir son propos aux maux de la société actuelle en général: violence, machisme, guerre, exploitation des femmes... Elle construit son propre langage, inventif et percutant pour se confronter à l'impasse du langage standardisé. Elle met donc en place «son propre arsenal linguistique où les mots semblent attaquer et contre-attaquer les limites de la syntaxe ${ }^{10} \gg$ (Peacock, 2015, p. 161).

En 2004, Born Bad a reçu le prix Laurence Olivier qui récompense les auteurs les plus prometteurs. Depuis, les pièces de $d$. tucker green ont été jouées sur des scènes prestigieuses telles que le National Theatre, le Hampstead Theatre, et par des compagnies telles que la Royal Shakespeare Company. Ses propres productions mettent en scène essentiellement des artistes noirs de diverses origines et fonctionnent comme un collectif. Ses textes sont écrits et proférés sur scène dans un langage original, complexe, expérimental, essentiellement poétique, tout en adoptant divers styles. La langue est fragmentée, parcourue de mots émergeants essentiels. Elle respecte un rythme qui s'apparente à une partition de jazz avec ses loops (boucles), repeats (reprises), interlocks (emboitements).

63 Elle s'inscrit dans une sphère identitaire spécifique, dans la temporalité d'un présent de plus en plus complexe et revendicatif. Elle use d'un style néo-réaliste poétique qui présente l'individu dans son contexte collectif pour mettre en mots et en scène une situation conflictuelle. Elle s'exprime dans une langue métissée très personnelle mais 
qui se rattache aussi à la tradition ancestrale de la communauté jamaïcaine. Elle se réfère aussi à la langue de la rue riche en inventions langagières et en rythmiques inventives. Chaque texte et chaque spectacle engagent une recherche formelle pour mettre en perspective un groupe humain particulier emblématique dans un contexte de crise de l'individu. Elle braque l'éclairage sur des groupes de population qui avaient été jusque-là largement méconnus ou négligés, voire discriminés par l'histoire et la littérature occidentales. Elle explore essentiellement les crises familiales d'un prolétariat malmené par les diktats de la hiérarchie sociale de nos sociétés ainsi que les crises politiques des sociétés contemporaines postcoloniales et pose la question de la responsabilité individuelle et collective.

L'univers de d. tucker green est très secret. Il habite la mémoire des gens ordinaires, des marginaux, des exclus dont elle explore les moindres recoins comme autant de lieux familiers. Elle observe, écoute les paroles et les chants qui lui parviennent d'un passé lointain, d'un territoire désormais étranger qu'elle redécouvre. Elle sait écouter les mots, entendre les paroles et les restituer par le corps et par la voix. Elle est la mémoire des déracinés, en particulier ceux de l'Empire Windrush, navire emblématique de l'exil caribéen, mais aussi celle de tous les « damnés de la terre " ${ }^{11}$.

Elle réécrit la nation britannique avec son histoire émaillée d'exils rarement volontaires mais... "London is the place to be", disait la chanson ${ }^{12}$ ! Se dessinent irrémédiablement la difficulté de vivre dans un environnement hostile, ainsi que la cruauté du destin pour ceux qui cachent leurs blessures et vivent en marge de l'histoire et en paient les dérapages.

Elle privilégie fréquemment des lieux plus intimes souvent non définis. Ce peut être le lieu de la famille: cuisine, chambre, salle commune où l'on peut s'affronter, où la parole se libère de manière souvent implicite, c'est-à-dire dont le sens se dévoile progressivement. Ce sont aussi parfois des mondes d'exclusion comme les pays en guerre. Ce peut être un ailleurs socialement et géographiquement neutre: la scène théâtrale où la confrontation devient plus politique et où la parole s'impose. Dans ses films, la rue ou la clairière est très présente. Ce sont les corps et les regards qui interrogent. L'espace est clos ou circonscrit et c'est l'espace de la scène - très épuré -, ou l'œil de la caméra - très proche - qui le rend explicite puisque ce qui importe c'est la relation à l'autre.

Elle scrute les conflits domestiques ou politiques qui opposent et relient les humains, et elle montre leur violence, leurs incompréhensions, leurs frustrations sans jamais juger. Elle met à nu la dureté de la vie qui exclut les plus démunis et les pousse à la marge (dirty butterfly). Elle dissèque les comportements humains et le relationnel sous l'influence d'environnements marginaux, cadres familiaux durement perturbés par des contextes politiques et sociaux destructeurs (stoning mary). Elle éclaire les actes barbares qui en résultent et met en exergue la situation des femmes surexploitées dans une société essentiellement machiste et cruelle comme dans sa pièce bad (mauvaise).

68 La parole de d.tucker green est calquée sur l'oralité de la rue, proche de celle des poètes emblématiques qui empruntent le phrasé de la langue jamaïcaine avec les particularités de leurs textes et de leurs rythmes musicaux. La rythmique très remarquable de sa parole théâtrale et la vivacité de la profération donnent voix aux thèmes qui hantent tout notre siècle - identité, pauvreté, inceste, guerre (enfantssoldats), racisme, féminisme... Son écriture passe de la prose à la versification et s'apparente également à une lignée d'auteures contemporaines par la recherche 
stylistique originale d'une langue spécifique elliptique et percutante (tucker green, 2018, p. 10) :

\section{US}

African Americans

\section{OLDER (WO)MAN}

Before the sun got cold (and) turn't the

skies to grey...

Before the wind got busy and rain decided

it couldn't be fucked to fall

Before the seasons decided they weren't

worth changing

we wasn't worth the effort

we weren't worth changing for.

before the green grass browned

before the concrete could warm

before the street lights repeat their on-off

on-off ${ }^{13}$.

\section{Conclusion}

\section{Représenter le réel sur scène}

D. Greig et d. tucker green, auteurs et dramaturges, cumulent les fonctions artistiques et techniques en se plaçant à la jonction de tous les rouages de la représentation théâtrale, de l'écriture à la réalisation. Après le théâtre total émerge l'artiste total. Se posent alors les questions de l'emprise de l'auteur sur son œuvre et du rapport à la scène et au public.

70 Si les œuvres de D. Greig circulent dans des mises en scène et dans des espaces multiples, la pièce représentée s'adapte par nécessité: le rapport au public est primordial, ce qui implique une réelle flexibilité. Chez D. Greig la fonction politicosociale prédomine, l'interrogation sur le présent interpelle le public frontalement.

71 Le théâtre de d. tucker green demeure essentiellement une expérience intime. La représentation s'apparente à une performance où les participants peuvent être interchangeables et anonymes puisque c'est la parole qui les façonne et leur témoignage qui importe.

72 Ces théâtres de l'engagement sont des espaces de désacralisation qui appellent à l'implication du spectateur que le spectacle interpelle comme témoin d'un monde qui se délite. La violence de l'époque y trouve son reflet.

\section{BIBLIOGRAPHIE}

AINSLIE, S. \& WARREN-FISCHER, R. (2010). Complicite: Rehearsal Notes; A visual essay of the unique working methods of the company. Londres : Éd. Complicite. 
BAUDRY, R. \& JUCHS, J.-P. (2007). « Définir l'identité ». Hypothèses, 1 p.155-167.

BLYTHE, A. (2011). London Road. Londres : N. Hern.

DAVID, G. (2006). « Portrait de l'Artiste Voyageur : Simon McBurney ou l'imaginaire en mouvement ». Études Théâtrales, 37, p.58-65.

MacColl E. (1990). Journeyman, an Autobiography. Londres : Sidgwick and Jackson.

MACKRELL, J. (2011). « Can we talk about this? - Review ». The Guardian. En ligne : https:// www.theguardian.com/stage/2011/nov/04/can-we-talk-about-this

MÉRAHI, D. (2010). Joan Littlewood l'insoumise et le Theatre Workshop. Montpellier : Éd. L'Entretemps.

MÉRAHI, D. (2017). Théâtres du réel en Angleterre et en Écosse depuis les années 1950. Paris :

Éd. L'Harmattan.

OLUSOGA, D. (2016). Black and British: A Forgotten History. Londres : MacMillan.

PAGET, D. (1990). True Stories? Documentary Drama on radio, screen, and stage. Manchester :

Manchester University Press.

PAVIS, P. (2019) [1980]. Dictionnaire du théâtre. Paris : A. Colin.

PAVIS, P. (1998). « Conférence Théorie et Pratique dans les études théâtrales, Monash University Juillet 1997 ». Théâtre, 1. En ligne : https://books.openedition.org/septentrion/13748?lang=fr

PEACOCK, K. (2015). « The Social and Political Context of Black British Theatre: the 2000s ». in :

Brewer, M. F., Goddard, L. \& Osborne, D. (eds), Modern and Contemporary Black British Drama.

Basingstoke: Palgrave Macmillan.

tucker green, d. (2018). Ear for eye, Londres : N. Hern.

WEISS, P. (1971). « The Material and the Models. Notes towards a definition of Documentary Theatre ».

Theatre Quarterly, 1 (1).

\section{NOTES}

1. Performance ou performance art serait plus proche de " théâtre des arts visuels ».

2. Provoke est un collectif japonais. Exposition à Le Bal, 6, impasse de la Défense, Paris $9^{\mathrm{e}}$, du 14 septembre au 11 décembre 2016.

3. Traduction D. Merahi.

4. Pièce créée en avril 2011 au Cottesloe, National Theatre à Londres.

5. Citations extraites du programme proposé au public lors des représentations de Can We Talk About this? Théâtre de la Ville à Paris du 28 septembre au 6 octobre 2011 (40 e édition du Festival d'Automne à Paris).

6. Le texte original du programme en anglais est le suivant : «...the interrelated issues of freedom of speech, multiculturalism and Islam as manifest in Western democracies... ».

7. Libre adaptation d'un texte de S. McBurney dans son introduction à Ainslie, s. \& Warren-Fischer, R. (2010).

8. Le designer M. Levine évoque le procédé de création des artistes cubistes pour définir les répétitions de la compagnie (Ainslie, s. \& Warren-Fischer, R., 2010). 
9. «En 1992, dans la pièce Mnemonic on devait représenter un cadavre vieux de 5000 ans qui émergeait tout noir tout ridé d'un glacier. Il est clair que toute représentation littérale aurait été plus que grotesque. Seuls les mots se référant à son apparence auraient une portée plus forte qu'un banal accessoire. Mais il fallait quelque chose pour le signifier. La chaise. La chaise était plus signifiante si elle était cassée. Alors on a utilisé une chaise cassée. » Traduction D. Mérahi.

10. "her creation of a linguistic arsenal by which words seem to attack and counter attack the bounds of syntax... ». Traduction D. Mérahi.

11. Voir livre de F. Fanon Les Damnés de la terre, Maspero, 1961.

12. Chanson citée par D. Olusoga: Black and British, a Forgotten History Pan Books, Paperback. An imprint of Pan MacMillan, 2017 p. 493. Le chanteur de calypso originaire de Trinidad Lord Kitchener (de son vrai nom A. Roberts) fut convié à interpréter cette chanson à l'arrivée du navire the Windrush à Tilbury docks, Londres le 22 juin 1948.

13. Avant que le soleil se refroidisse (et) que les cieux soient gris/Avant que le vent s'active et que la pluie décide/ qu'elle n'allait pas se faire chier à tomber/Avant que les saisons décident que ça ne valait pas la peine de changer/qu'on n'en valait pas l'effort/ qu'on n'en valait pas la peine/avant que l'herbe verte brunisse/avant que le béton se réchauffe/ avant que les lumières de la rue s'allument et s'éteignent/s'allument et s'éteignent. Traduction D. Mérahi.

\section{RÉSUMÉS}

Les théâtres du réel sont issus d'une longue tradition anglaise dans le théâtre britannique. Ils traitent des réalités sociales et politiques du présent et offrent un large éventail d'esthétiques. Ils caractérisent les complexités d'une époque où le créateur et le spectateur ont du mal à trouver un éventuel terrain d'entente.

On parle de théâtre documentaire, de chronique historique, de théâtre politique, de théâtre verbatim, de performance, de stand up... L'utilisation d'autant de formes dramatiques reflète la dimension d'une réflexion critique nationale. La distance entre fiction et réalité devient de l'ordre de la responsabilité du spectateur. Les théâtres du réel s'intéressent aux groupes ou aux communautés marginales qui prennent la parole, aux minorités ethniques qui inventent et imposent leur propre langage.

Ma contribution ne peut se vanter d'avoir trouvé une réponse définitive pour définir les questions artistiques soulevées par tant de créations multinationales pleines d'imagination: leurs approches politiques sont déployées par tant de groupes artistiques activistes désireux d'exprimer les réalités de notre temps, qu'il est fascinant de mesurer la richesse des recherches créatives qui prévalent dans le monde artistique.

Theatres of the Real stem from a long-running tradition of « Englishness » in the British theatre. They appear under several aspects dealing with the social and political realities of the present and offer a wide range of aesthetics. They characterize the complexities of the times when the creator and the spectator may experience difficulties to find a potential common ground. Various expressions are privileged such as documentary theatre, historical chronicle, political 
theatre, verbatim theatre, performance, stand up... In other words, the use of so many dramatic forms assume the dimension of a national critical reflection. The distance between fiction and reality becomes the responsibility of the spectator. Theatres of the Real care for marginal groups or communities who speak up, ethnic minorities who invent and impose their own language.

My essay cannot boast about having found a final answer to define the artistic issues raised by so many imaginative multinational creations. Their political approaches are submitted by many activist artistic groups willing to express the realities of our time. Yet it is fascinating to take note of the rich creative pursuits that prevail in the artistic world.

\section{INDEX}

Keywords : contemporary theatre, real, realism, British theatre, politics, social, local, commitment

Mots-clés : théâtre contemporain, réel, réalisme, théâtre britannique, politique, social, local, engagement 